\title{
Detector Performance Analysis of Coverage Area Difference movement in Sensor Network Based on Triangulation
}

\author{
Hai Qiao ${ }^{1}$, Shi Feng ${ }^{1}$
}

${ }^{1}$ Department of physical Education, Nanchang Institute of Technology, Nanchang, 330099, China

hai_qiao@yeah.net

Keywords: Feeling difference, Wireless sensor, Progressive scan, Mobile node, Target coverage, Muscle displacement.

\begin{abstract}
In order to study effects of badminton players smashing training on proprioceptive features, explore proprioceptive features effect on fine motion control of the body and reveal the brain producing mechanism, this paper uses progressive scan mode and wireless sensor device to design the sensor nodes of motion mode, and 2 nodes covering 3 goals. Using the muscle activity 3D space as the goal, the sensor node is discredited. Through the measurement of the badminton players muscle displacement, this paper obtained the characteristic differences of body movement, which provides reliable data library for badminton players spiking training.
\end{abstract}

\section{Introduction}

Definition of proprioception was originally proposed by Charles Bell, he made proprioception as the fundamental structural basis of perception and motion, while the modern scholars see the proprioception as special variables of measuring kinesthetic and joint position [1, 2]. This paper uses wireless sensor device and mobile sensor node distribution to analyze the muscle displacement changes of player in the movement process, so as to obtain the best action points of spiking in the process. The main process is as follows.

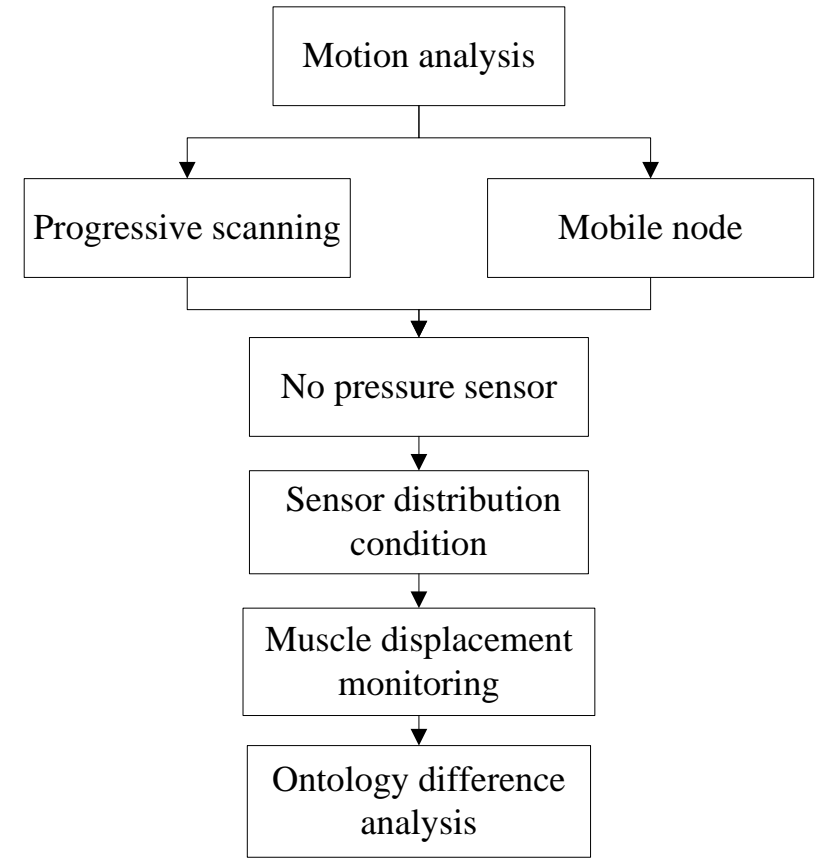

Fig.1: Detection of badminton smashes body difference

Figure 1 shows the overall design framework of wireless sensor. This paper adopts a 2D sensor line by line scanning principle and uses mobile sensor nodes to monitor muscle displacement of badminton players spiking [3]. The collected data is transmitted to the data processing center, by marking the best buckle ball point, so it can provide reliable data for the badminton athlete's training material. 


\section{Design of Body Motion Sensation Sensor Coverage Model}

In order to accurately measure the proprioception difference in the process of badminton smash, it uses more advanced sensor to cover networks to scan in multi angles and full range [4-6]. The scanning process is as shown in Figure 2.



Fig.2: 2D sensor scanning model

As shown in Figure 2 it is the schematic diagram of $2 \mathrm{D}$ scanning sensor model, and the coordinate is $C(x, y, z)$, the sensing radius is $R$, then the perceived direction is $D=(d x, d y, d z)$. It uses mobile nodes to realize the sensor full coverage in the process of badminton smashing.

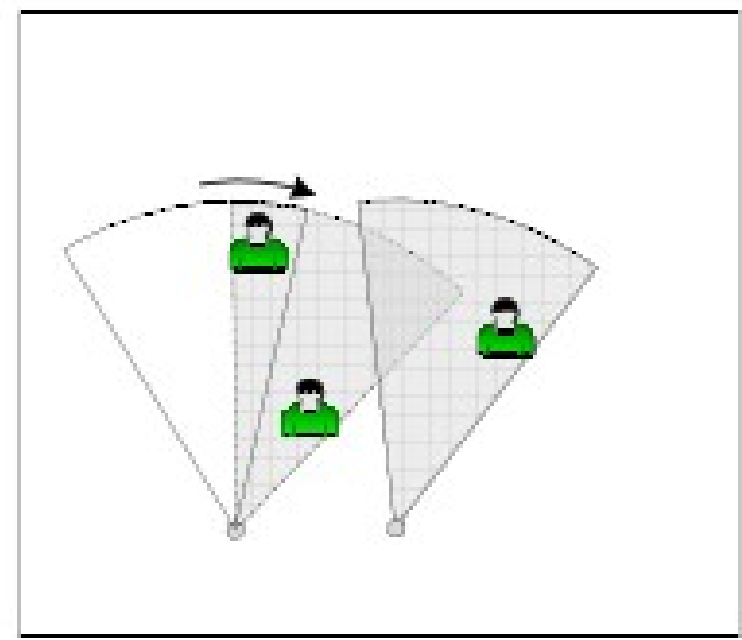

Fig.3: The mobile node coverage diagram

Through the node movement, Figure 3 uses 2 nodes to cover 3 targets [7]. Using the 3D space as the example, it uses the method of progressive scanning, to discrete sensor nodes as shown in formula (1).

$$
u_{i, j, k}^{n}=u(t, x, y, z)_{t=n \Delta t, x=i \Delta x, y=j \Delta y, z=k \Delta z} .
$$

Among then, $u_{i, j, k}^{n}$ shows the node function; $x, y$ and $z$ indicate the position coordinates of three directions; $u(t, x, y, z)$ is displacement of three directions. For the first-order partial derivatives, it uses Euler's formula to obtain the forward Euler formula. 


$$
\begin{aligned}
& \left.\frac{\partial u}{\partial t}\right|_{t=n \Delta t, x=i \Delta x, y=j \Delta y, z=k \Delta z}=\frac{u_{i, j, k}^{n+1}-u_{i, j, k}^{n}}{\Delta t} \\
& \left.\frac{\partial u}{\partial x}\right|_{t=n \Delta t, x=i \Delta x, y=j \Delta y, z=k \Delta z}=\frac{u_{i+1, j, k}^{n}-u_{i, j, k}^{n}}{\Delta x} \\
& \left.\frac{\partial u}{\partial y}\right|_{t=n \Delta t, x=i \Delta x, y=j \Delta y, z=k \Delta z}=\frac{u_{i, j+1, k}^{n}-u_{i, j, k}^{n}}{\Delta y} \\
& \left.\frac{\partial u}{\partial z}\right|_{t=n \Delta t, x=i \Delta x, y=j \Delta y, z=k \Delta z}=\frac{u_{i, j, k+1}^{n}-u_{i, j, k}^{n}}{\Delta x}
\end{aligned}
$$

For the two order partial derivatives, it can use the Taylor expansion processing technology to obtain the following discrete calculation formula:

$$
\begin{aligned}
& \left.\frac{\partial^{2} u}{\partial t^{2}}\right|_{t=n \Delta t, x=i \Delta x, y=j \Delta y, z=k \Delta z}=\frac{u_{i, j, k}^{n+1}-2 u_{i, j, k}^{n}+u_{i, j, k}^{n-1}}{\Delta t} \\
& \left.\frac{\partial^{2} u}{\partial x^{2}}\right|_{t=n \Delta t, x=i \Delta x, y=j \Delta y, z=k \Delta z}=\frac{u_{i+1, j, k}^{n}-2 u_{i, j, k}^{n}+u_{i-1, j, k}^{n}}{(\Delta x)^{2}} \\
& \left.\frac{\partial^{2} u}{\partial y^{2}}\right|_{t=n \Delta t, x=i \Delta x, y=j \Delta y, z=k \Delta z}=\frac{u_{i, j+1, k}^{n}-2 u_{i, j, k}^{n}+u_{i-1, j, k}^{n}}{(\Delta y)^{2}} \\
& \left.\frac{\partial^{2} u}{\partial z^{2}}\right|_{t=n \Delta t, x=i \Delta x, y=j \Delta y, z=k \Delta z}=\frac{u_{i, j, k+1}^{n}-2 u_{i, j, k}^{n}+u_{i, j, k-1}^{n}}{(\Delta z)^{2}}
\end{aligned}
$$

Using super relaxation iteration, it can accelerate progressive scanning.

$$
\left\{\begin{array}{c}
\bar{u}=\frac{1}{4}\left(u_{i+1, j}^{(k)}+u_{i, j+1}^{(k)}+u_{i-1, j}^{(k+1)}+u_{i, j-1}^{(k+1)}\right) \\
u_{i, j}^{(k+1)}=w \bar{u}+(1-w) u_{i, j}^{(k)}
\end{array} .\right.
$$

Progressive scan results of mobile sensors can be written as a linear equation form.

$$
C X=Y \text {. }
$$

In order to realize the sensor measure on the badminton athletes body difference, this paper collects data of ground pressure and muscle displacement in the athletic motion process, and the algorithm is as follows:

void osalAddTasks( void )

\{osalTaskAdd(PresureSensor_Init,PresureSensor_ProcessEvent,OSAL_TASK_PRIORITY_LO W);

\}

osalTaskAdd

PresureSensor_Init

Apus_TaskID = task_id;

PresureSensor_epDesc.endPoint = APUS_ENDPOINT;

PresureSensor_epDesc.task_id = \&Apus_TaskID;

PresureSensor_epDesc.simpleDesc

$=($ SimpleDescriptionFormat_t $*) \&$ Apus_SimpleDesc;

PresureSensor_epDesc.latencyReq = noLatencyReqs; 


\section{Study on the Wireless Sensor Measurement of Badminton Smash Body Differences}

In order to effectively verify the effectiveness and reliability of mobile sensor progressive scanning mathematical model, this paper designs the sensor measurement system of badminton smash ontology difference [8-10]. The system consists of sensor node, routing node, sink node, local monitoring center and the remote monitoring center. Its structure is as shown in Figure 4.

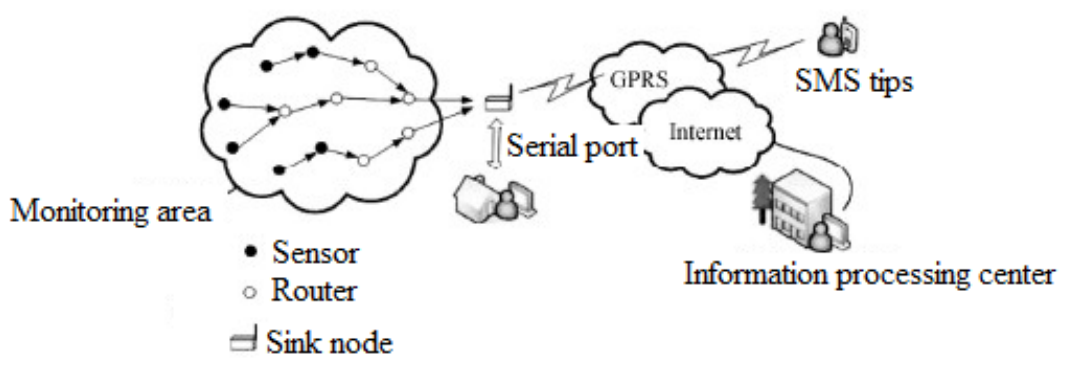

Fig.4: The monitoring system structure

As shown in Figure 4, the sensor node and routing nodes deployed in the monitoring region, forming a self - organization network [11]. The collected data is transmitting to the sink node in the form of multi hop, through the RS23 serial port the data is transferred to a local monitoring center, by processing the muscle displacement data, the data of body differences is obtained.



Fig.5: Schematic diagram of node package

Figure 5 shows the schematic diagram of the node package. The processor module uses TI's single chip MSP430F149, and the most prominent feature of the chip is low voltage, low power consumption, suitable for low power consumption occasions. The wireless communication module uses nRF905 module with low power consumption and adjustable transmitting power, and the transmission distance is long, anti-interference ability is strong [12]. The inner-builtidle mode and off mode is easy to realize.

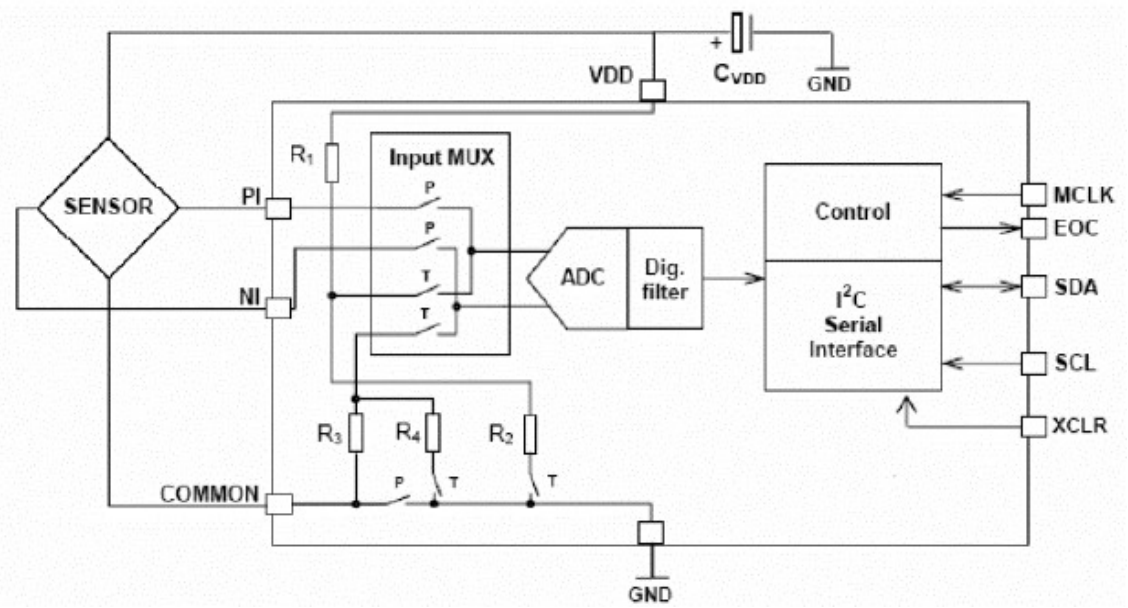

Fig.6: Block diagram of HP03D pressure sensor internal 
Figure 6 shows the circuit diagram of the pressure sensor [13]. The internal of HP03 has a pressure electric resistance and a 15 bit A/D converter, the output interface is the standard interface, internal storage 11 correction parameters, so as to correct the measuring results.

\begin{tabular}{|c|c|c|c|c|c|}
\hline P.nbr. & Time (ms) & \multirow{2}{*}{ Length } & \multicolumn{2}{|c|}{ Frame control field } & \multirow{2}{*}{$\begin{array}{l}\text { Sequence } \\
\text { number }\end{array}$} \\
\hline$R X$ & +3 & & Tyoe Sec Pnd & ack, ced PAग_congr & \\
\hline 64 & $=11167$ & 27 & CMD & 1 & $0 \times 31$ \\
\hline P.nbr. & Time (ms) & $1 \operatorname{sn} \pi$ & \multicolumn{2}{|c|}{ Frame control field } & Sequence \\
\hline $\mathrm{RK}$ & +113 & Lengin & Trpe जేec Fnd & Ack. Ieq PaN_conpL & number \\
\hline 66 & $=11282$ & 51 & DдТА 0 & 1 & $0 \times 32$ \\
\hline
\end{tabular}

Fig.7: Address allocation and the routing topology

Figure 7 shows the test results of address allocation and routing topology. By testing, terminal equipment can connect the routers after joining the network, and then it is assigned a short address [14]. The router reports the network topology changes to coordinator, so as to test the body difference of badminton smash process.



Fig.8: The orientation of body differences

In order to study on the body difference of badminton smash training, through mobile nodes coverage measurement, this paper uses wireless sensor to record two times spike, and the muscle displacement difference curve is obtained [15]. From the chart it can be seen, the muscle displacement is significantly different in the two spike training, so they need repeated training and measurement, \ finally the best muscle displacement curve of spike can be obtained, and data is marked using the data processing center, which provides the database material for badminton training.

\section{Summary}

Combined with the Taylor formula, this paper uses the first order partial derivatives and two order partial derivative principles to establish the mathematical model of sensor 3D progressive scanning, and designs mobile wireless sensor network nodes, which improves the coverage of wireless sensor network. Using the sensor node discrimination, this paper measures the 3D characteristics of badminton athlete training muscle, and the body characteristic differences of muscle displacement is obtained, which provides data reference and theoretical basis for the badminton players spiking training. The system can be extended to other project training, but also can be applied to other sports fields, which can provide technical support for the training of athletes.

\section{References}

[1] S. Gao, H.K. Zhang. Delay constrained mobile Sink sensor network path selection method. Acta electronica Sinica, 2012, 3(4): 56-59.

[2] S. Gao, H.K. Zhang, H.S. Xu. Efficient data collection mechanism of Sink trajectory fixed sensor networks. Journal of software, 2012, 4(1): 24-28.

[3] S. Jiang, W.X. Wang, D.Z. Sun, Z. Li. Wireless sensor network node design of energy selfsufficient orchard information gathering. Journal of agricultural engineering, 2012, 3(9): 78-81.

[4] W.X. Wang, X.W. Luo, D.Z. Sun, S. Jiang, G.K. Xiao. Wireless sensor network node design of tea information collection. Journal of agricultural engineering, 2013, 3(5): 34-38. 
[5] S. Zou, C.M. Liu, F.P. Li. Water environment monitoring system based on wireless sensor network sensor. Sensor and micro system, 2012, 4(9): 81-84.

[6] C.F. Wan, S.F. Du, L. Zhao. Research on sinus aquaculture environment monitoring system based on WSN. Agricultural machinery, 2012, 4(2): 45-48.

[7] M.D. Xue. Temperature and humidity measurement system with low power consumption based on nRF905. Modern electronic technique, 2012, 4(1): 91-95.

[8] D.M. Fan. Analysis of life time physical education and lifelong physical education. Examination weekly, 2013, 4(4): 12-16.

[9] J.C. Jia. Talk about badminton and fitness exercise. Union Forum (Journal of Shandong Institute of Trade Union Cadres), 2013, 4(5): 45-48.

[10] L.Y. Shen. The college badminton teaching and lifelong physical habits. Science Journal, 2012, 3(8): 23-26.

[11] J.T. Shao. Motivation study of masses intersted in badminton sport. Information technology. 2012, 3(8): 78-80.

[12] J. Wang, H. Chen. Present situation investigation and development countermeasure research of the Guangzhou city mass badminton place service. Journal of Guangzhou Sports University, 2012 3(1): 34-37.

[13] F. Xin. The current situation and optimization of college PE teaching model. Hubei sports science and technology, 2012, 3(5): 34-38.

[14] X.Z. Qi. Research on traditional sports teaching mode and modern sports teaching mode. Sports world (Academy version), 2012, 3(4): 12-15.

[15] J.J. Xu. The value significance of badminton sport. Science and technology information, 2012, 3(30): 45-48. 